\title{
The Develop of an Automatic Test System for the Carbone Controller
}

\author{
Dandan Liu ${ }^{1,2}$, Xiangbing $\mathrm{Qi}^{3}$ and Xiangxian Chen ${ }^{1+}$ \\ ${ }^{1}$ College of Biomedical Engineering \& Instrument Science, Zhejiang University, Hangzhou 310027, \\ China \\ ${ }^{2}$ Zhejiang Zheda Train Intelligent Engineering Technology Research Center Co., Ltd Hangzhou \\ 310051, China \\ ${ }^{3}$ United Science \&Technology Co., Ltd., Research and Development Center, Hangzhou 310051, China
}

\begin{abstract}
This paper introduces an automatic testing system for the carbone controller. The detailed analysis is carried out around hardware and software design. An automatic test system is developed, and is verified by taking BiCC carbone controller as an example. Practice shows that the automatic test system plays an important role in the testing process of the factory and meets the requirements of the factory test.
\end{abstract}

Keywords: automatic test system, carbone controller, factory test

\section{Introduction}

Carbone controller is an important part of urban rail transit signal system, which involves operational safety of the train and passenger comfort [1]. Factory test is the last test operation before delivering to the user, the purpose of which is to ensure that the software and hardware versions of the whole set are consistent, the whole set functions normally and meets the system requirements [2].

Because there are many interfaces between the carbone controller and the vehicle[3], and the peripherals vary from project to project, it is not realistic for the carbone controller to verify its correctness on the field verification. Therefore, factory test of the carbone controller needs a set of test system to support the verification in the factory environment [4], [5].

This paper develops an automatic test system, in which non-real-time related input and output interface signals are configured with digital input and output cards through the industrial control computer, while real-time related device interface signals use high performance embedded processors and real-time operating systems. The config file is read automatically and the test cases are run automatically. The test results are judged automatically by comparing the running results with the business simulation software in the carbone controller system, and the test report is output automatically.

\section{Hardware Structure of the Test System}

The automatic test system for the whole set mainly includes: simulation chassis, industrial control computer, switch and power module, among which the simulation cage includes a simulation main control board, a simulation input board and a simulation output board. The physical interfaces of carbone controller mainly include: input interface, output interface, analog interface, radar interface, speed sensor interface, network communication interface and power interface. The frame of the automatic test system is shown in Figure 1.

\footnotetext{
+ Corresponding author. Tel.: + 86-571-87953941; fax: +86-571-87953941.
}

E-mail address: xxchen99@zju.edu.cn. 
The IO interface of vehicle is a non-real-time signal, which configuring PCI digital input and output card through industrial control computer to directly collect output of the carbone controller and control input of the carbone controller. Because the interface signal of PCI digital input and output card is TTL electrical level, which is inconsistent with the electrical level of IO interface of the carbone controller, it connects with the carbone controller after the IO signal is adjusted by simulation input and output board. The IO signal of the main and standby system of the vehicle controller needs to synchronize with collection and output of the PCI digital input and output card, which can be realized through the simulation input and output board.

The interface signals of vehicle management system, radar sensor, speed sensor and beacon query machine are real-time signals. The signals of these interfaces are generated through the simulation master board, to ensure the real-time performance of the signals between the test system and the vehicle controller.

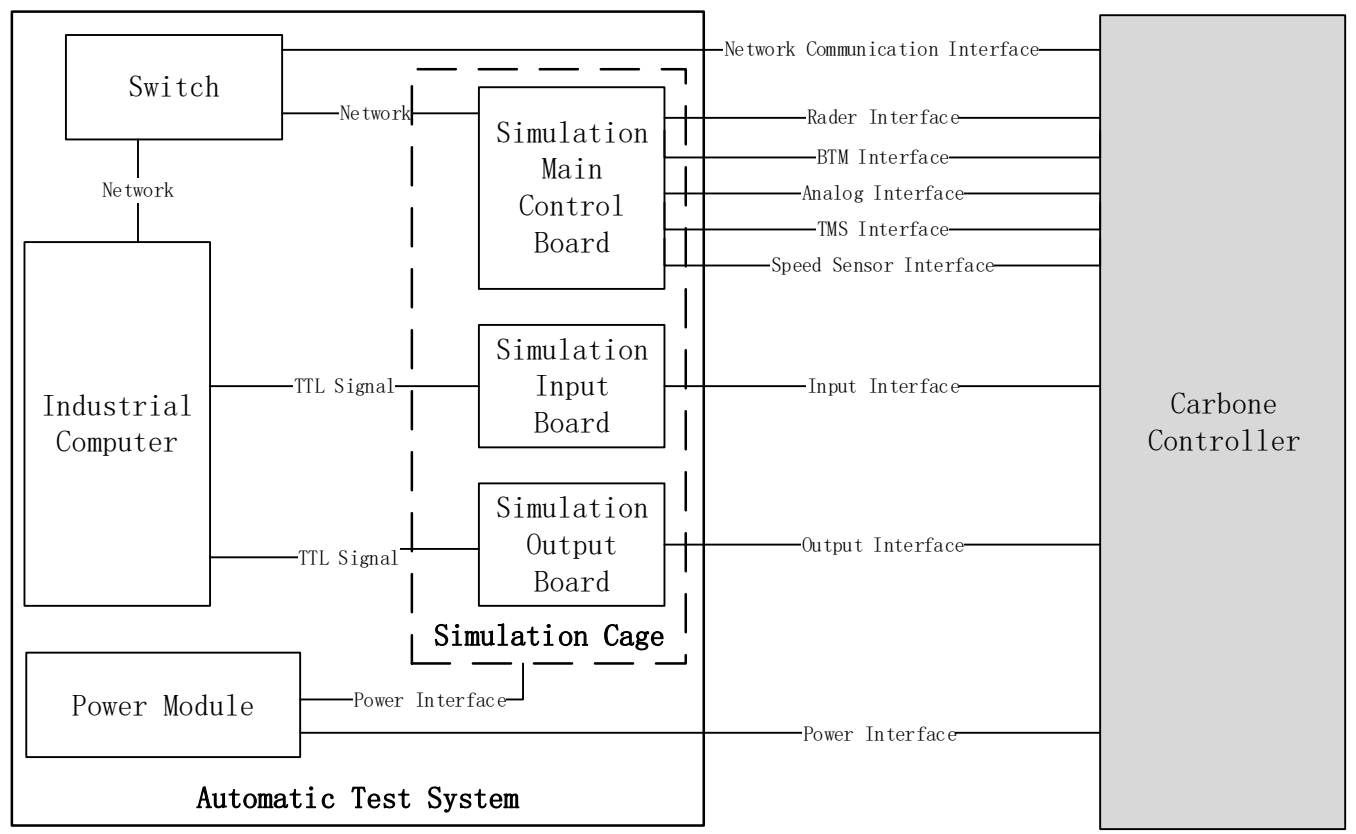

Fig. 1: Frame of automatic test system

\section{Software Design}

The automatic test system software include the IO simulation program ,CC business simulation softwareand whole set test software, which are deployed and run the test system on the industrial control computer and the carbone controller respectively. The flow chart of the software is shown in Figure 2.

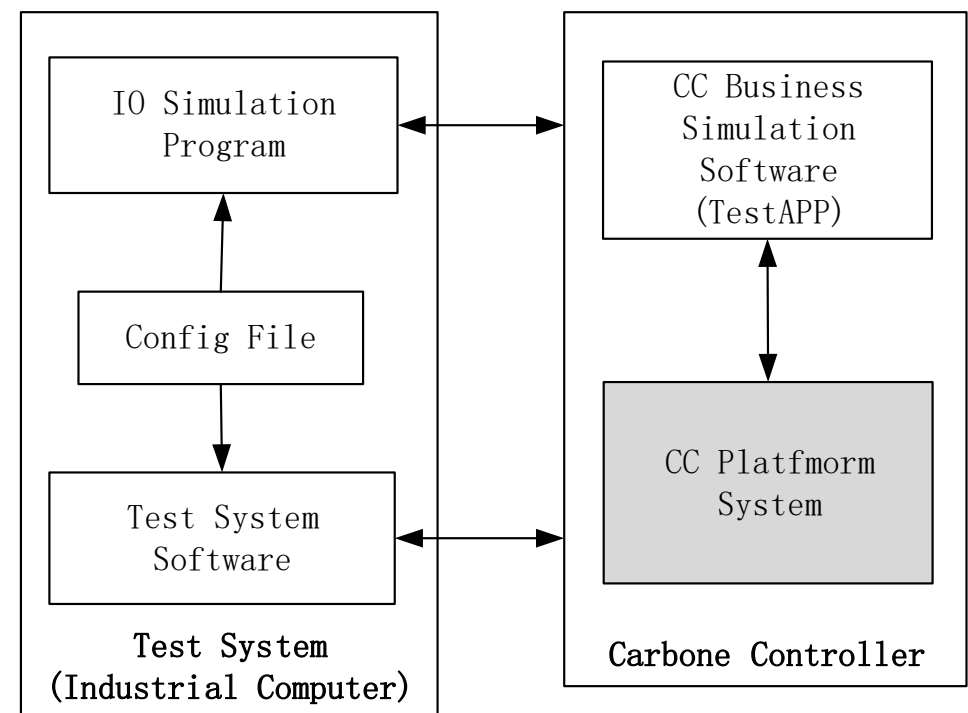

Fig. 2: The flow chart of automatic testing system software 
Based on the test system hardware and carbone controller platform system, the automatic test system for whole set delivery is used to control operation of the carbone controller platform, so that the config file is loaded and analyzed, the test command is issued, the test command is executed and the test result is display and saved, as well as realization of other functions. Functional data processing is shown in figure 3.

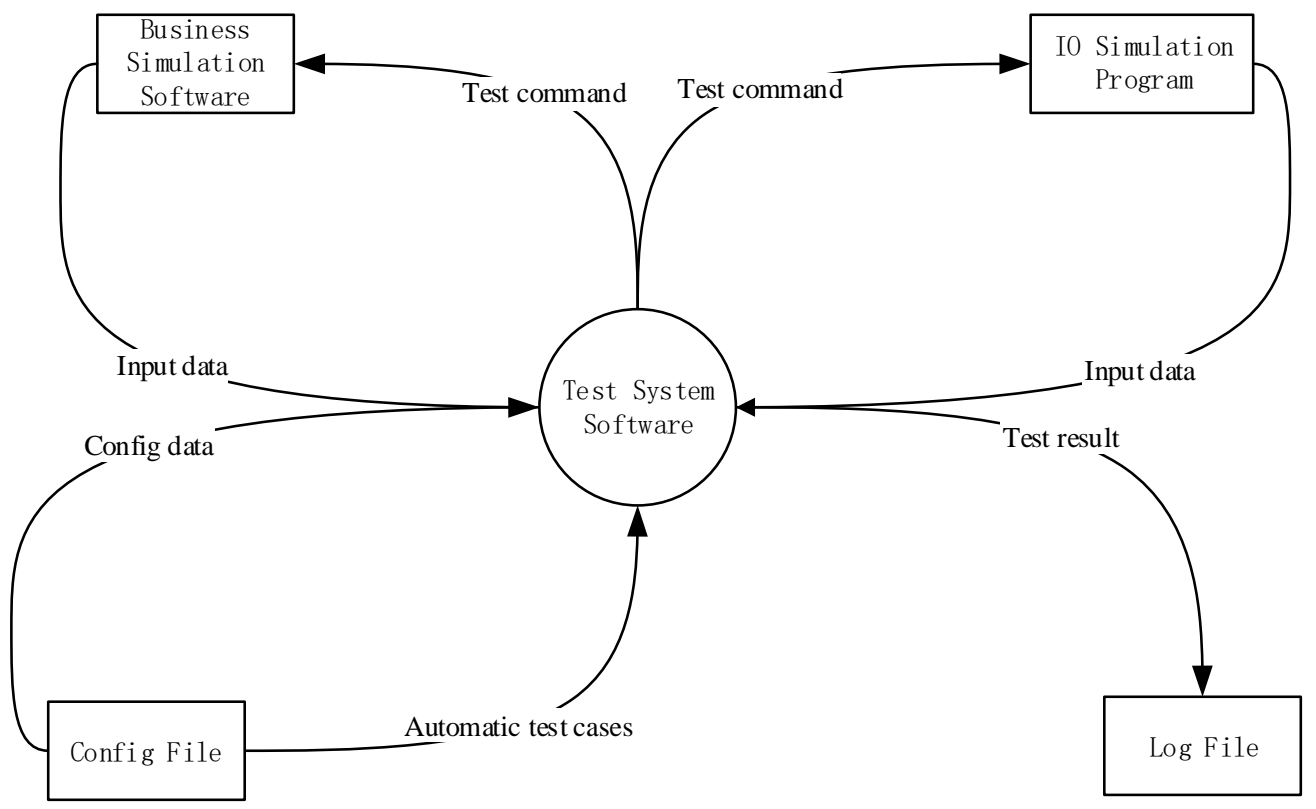

Fig. 3: Top layer data flow diagram of the automatic test system

\subsection{IO Simulation Operation Program}

The IO simulation operation program runs on the industrial control computer of the automatic test system, which is used to collect IO level signal of simulation input board and output IO level signal through simulation output board. Through IO simulation program, the whole testing software sends the IO status to the platform which transmits the status to the business simulation software in the form of message.

On the other hand, after the operation order of external equipment is sent to the platform software by IO command, which is converted into a level signal by the platform system, and is collected by IO simulation program and sent to the testing software of the automatic testing system software.

IO simulation operation program model is shown in figure 4.

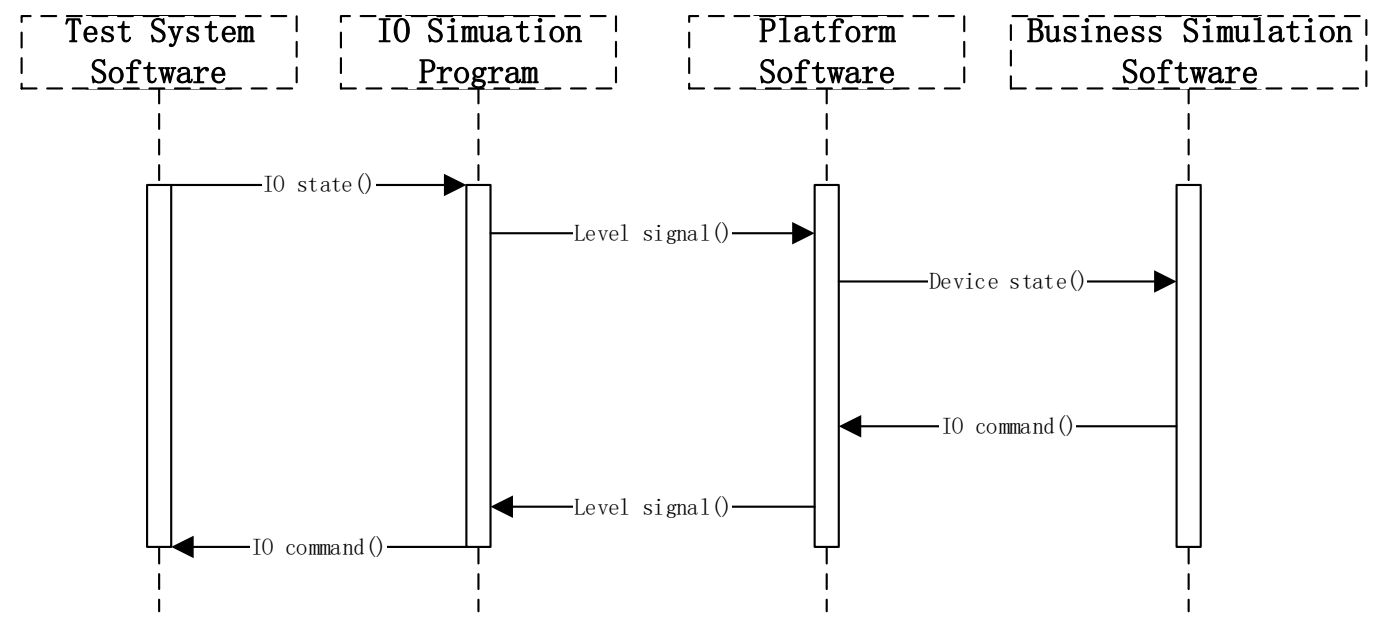

Figure 4: IO simulation operation program model

\subsection{Business Simulation Software}

The business simulation software (TestAPP) runs on the business software layer, context of which is shown in Figure 5. 


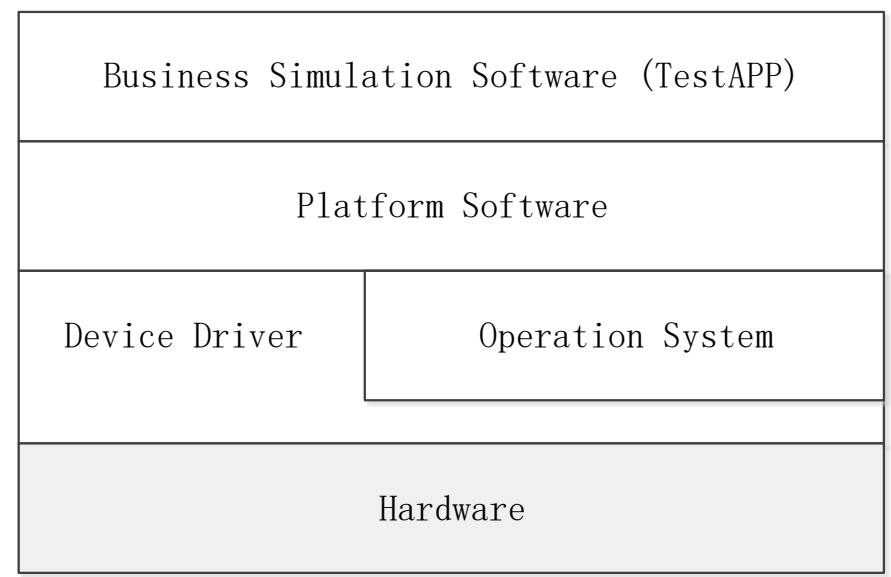

Figure 5: Context of the business simulation software

Platform software and business simulation software run in the same process and adopt API mutual calling mode during running, which use the same address space and single task running. The platform invokes the business simulation software initialization function, which is App_Init function during initialization phase, and invokes App_Loop function of the business simulation software in the main loop. The two functions return 0 to indicate the end of a full call, a positive number to indicate the need to be called again, and a negative number to indicate an error in the call. This kind of return design is to ensure that the running time from each call to return is no more than one maximum in a real time demanding system.

The business simulation software model is shown in figure 6 .

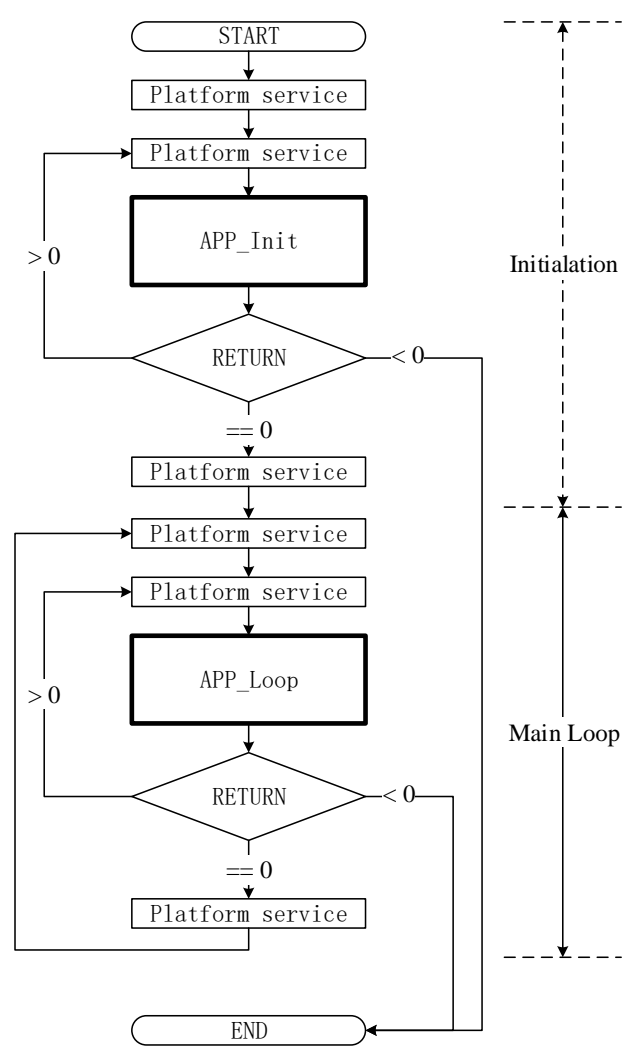

Fig. 6: Business simulation software model

\subsection{Whole Set Test Software}

The whole set test software includes config reading, analysistestcases, commands executing, log recording and other functions. The top layer data flow chart of the whole set test software is shown in figure 7. 


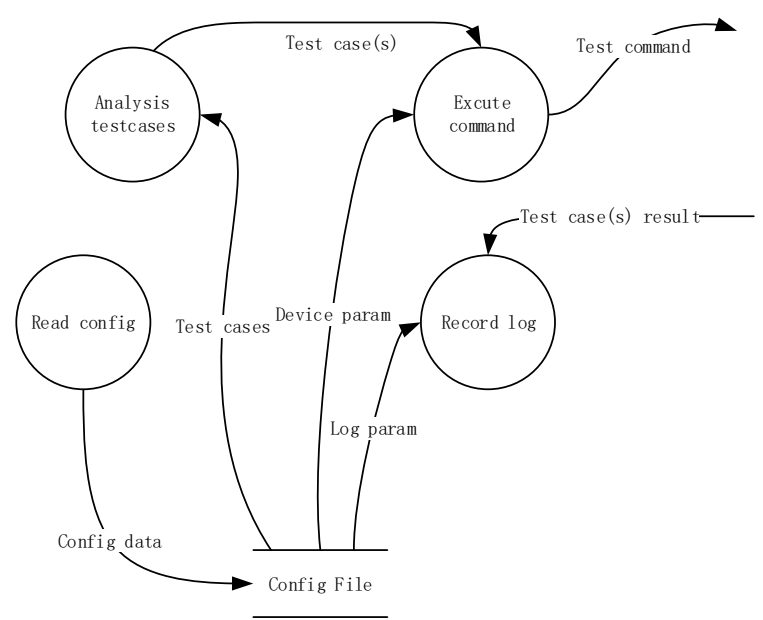

Fig. 7: Top layer data flow chart of the whole set test software

The config file is in $\mathrm{xml}$ format, the root node name of each test case is TestCase, and the child node is Step. Some examples of the config file test case are shown in figure 7.

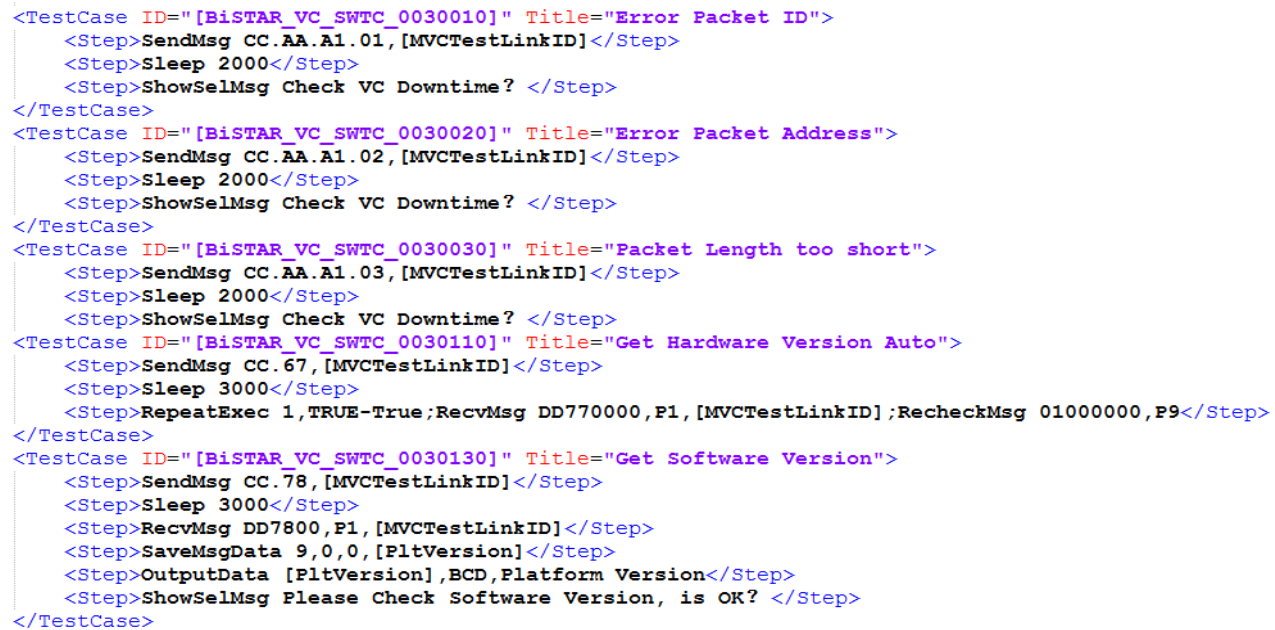

Fig. 8: Examples of the test case format for the config file

The UI interface of the whole set test software includes menu bar, showing the number of successful and failed use cases, real-time log window, etc. The user case execution interface is shown in figure 9.

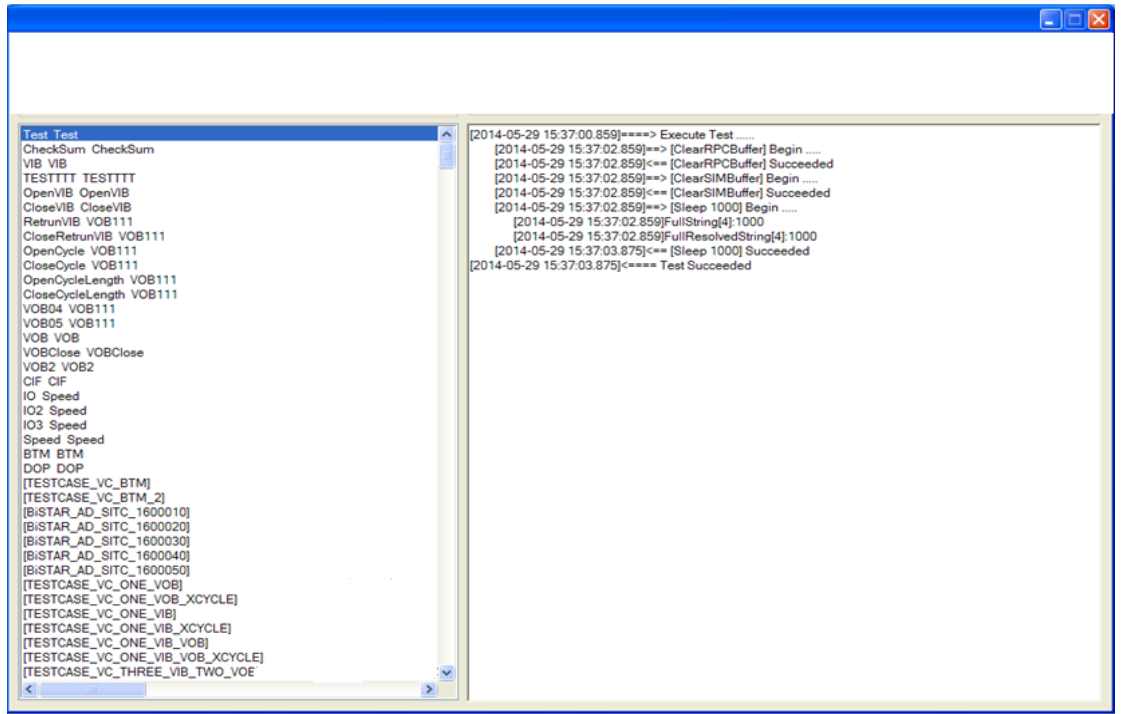

Fig. 9: Use case execution interface 


\section{Application of the Test System}

Taking the BiCC carbone controller as an example, the accuracy, integrity and practicability of the automatic test system is verified. The complete test function requirement table is shown in the table 1 .

Table 1: The complete test function requirement table of BiCC carbone controller

\begin{tabular}{|c|c|c|c|c|}
\hline Order & Function Requirement & Parameter & Type & Result \\
\hline 1 & ATP output test & $\begin{array}{l}\text { Output switching data } \\
\text { Recovery switching data }\end{array}$ & UINT64; UINT64 & \\
\hline 2 & ATO output test & $\begin{array}{l}\text { Output switching data } \\
\text { Recovery switching data }\end{array}$ & UINT64; UINT64 & \\
\hline 3 & ATP input test & $\begin{array}{l}\text { Output switching data } \\
\text { Recovery switching data }\end{array}$ & UINT64; UINT64 & \\
\hline 4 & $\begin{array}{l}\text { Speedometer interface } \\
\text { test }\end{array}$ & $\begin{array}{l}\text { Frequency value } \\
\text { Pulse number }\end{array}$ & UINT32; UINT64 & \\
\hline 5 & Radar interface test & $\begin{array}{l}\text { message } \\
\text { Frequency value }\end{array}$ & CHAR; UINT32 & \\
\hline 6 & TMS interface test & message & CHAR & \\
\hline 7 & $\begin{array}{l}\text { Current loop interface } \\
\text { test }\end{array}$ & Percentage value & UINT32 & \\
\hline 8 & Beacon interface test & $\begin{array}{l}\text { Message } \\
\text { Switching data }\end{array}$ & CHAR; UINT32 & \\
\hline 9 & Light and knob test & $\begin{array}{l}\text { Iutput switching data } \\
\text { Output switching data }\end{array}$ & UINT32; UINT32 & \\
\hline 10 & $\begin{array}{l}\text { Service synchronous } \\
\text { message test }\end{array}$ & Message & CHAR & \\
\hline
\end{tabular}

According to the automatic test records of the BiCC carbone controller for nearly a year, the system is stable and reliable. It takes about 15 minutes to test the whole equipment, while the original manual test time is about 3-5 hours. The test time are greatly saved, the test efficiency the consistency and the test quality is greatly improved.

\section{Conclusion}

The automatic test system for the carbone controller introduced in this paper is proved to be stable and reliable, which can completely meet the requirements of the automatic test for whole set delivery of the carbone controller. The test efficiency is improved and the test quality is effectively ensured. In addition, the system can be used for the factory test and the laboratory simulation test [6] [7] [8].

\section{Acknowledgement}

This work is supported by the funding from the National Science and Technology Infrastructure Program of China under Grant 2015BAG19B03

This work is supported by the funding from National Science and Technology support Plan of China under Grant 2015BAG13B01

\section{References}

[1] IEEE Vehicular Technology Society, "IEEE Recommended Practice for Communications-Based Train Control 
(CBTC) System Design and Functional Allocations”, IEEE Std 1474.3-2008, pp. 9-83, 2008.

[2] Pengfei Dai, Development of automatic testing tools for ATP/ATO on-board equipment[J], Railway Signalling \& Communication Engineering2017(05):66_68,78

[3] IEEE Vehicular Technology Society, "IEEE Standard for Communications-Based Train Control (CBTC) Performance and Functional Requirements”, IEEE Std 1474.1-2004, pp. 6-10, 2004.

[4] Daqing Wang, Acceptance Testing for Urban Rail Transit CBTC System[J],Urban Mass Transit,2016(3),21-27

[5] Yin Liming, Liu Junyan, Cheng Jia. HILS for the Software Development of On-board CBTC [J], Urban Mass Transit, 2011(04):40-44.

[6] Chao Wang, Research and implementation of Train Simulation Control system[J],Railway Signalling \& Communication,2016(04):51-53

[7] Wang Dong. Research and Application on the Simulation Testing and Verification Techniques in the Railway Signaling System. [D]. Zhejiang University. 2014.

[8] Zhang Kai, Feng Hu, Liao Xianghuang. Simulation and Detection System for Urban Rail Transition-board ATP Equipment [J]. Urban Mass Transit, 2012(03):61-64.

[9] Xu Qi-jun,Cao jian. Development of Intelligent Testing System for Gear Pump[J]. Chinese Hydraulics \& Pneumatics,2018(01):116-120 